\title{
Envenoming by the rough-scaled snake (Tropidechis carinatus): a series of confirmed cases
}

\author{
Melissa Gan, Margaret A O'Leary, Simon G A Brown, Tamara Jacoby, David Spain, Alan Tankel, \\ Chris Gavaghan, Peter Garrett and Geoffrey K Isbister
}

$\mathrm{T}$ he Australian snake Tropidechis carinatus (the "rough-scaled snake" [RSS]) (Box 1) is a relatively small snake (growing to about $1 \mathrm{~m}$ in length) that is taxonomically related to the Australian tiger snake (Notechis spp). The RSS and tiger snake are both "elapid" snakes (members of the family Elapidae), characterised by venom-secreting fangs that are fixed at the front of the upper jaw. The RSS inhabits temperate and tropical coastal regions and has previously been identified between northern New South Wales and south-eastern Queensland, with a few specimens found further north in Queensland. ${ }^{1,2}$ Features of envenoming are thought to be similar to tiger snake envenoming, including venom-induced consumption coagulopathy (VICC), myotoxicity and neurotoxicity. Snake venom detection kit (SVDK) testing for RSS venom gives a positive result for both RSS and tiger snake venom. ${ }^{3}$

Until recently, when an enzyme immunoassay (EIA) technique became available, it was not possible to confirm RSS envenoming unless a snake was provided for identification. Previous reports have been from snake handlers and botanists ${ }^{2}$ and may not be representative of the general patient population being bitten by snakes. Furthermore, in northern NSW and south-eastern Queensland, within the natural range of the RSS, it remains unclear what proportion of "tiger snake group" envenomings are due to RSSs. As well as tiger snakes, two Hoplocephalus species (Stephens' banded snake and the pale-headed snake) occur in the same region and their venoms may also test positive for tiger snake venom with SVDK.
Abbreviations
ASP Australian Snakebite Project
EIA Enzyme immunoassay
INR International normalised ratio
IQR Interquartile range
RSS Rough-scaled snake
SVDK Snake venom detection kit
VICC Venom-induced consumption coagulopathy

\section{ABSTRACT}

Objective: To describe demographic, geographical and clinical features of envenoming by the rough-scaled snake (RSS) (Tropidechis carinatus).

Design, setting and participants: Prospective cohort study of RSS snakebite victims, recruited between January 2004 and December 2008, as part of the Australian Snakebite Project. RSS envenoming cases were confirmed by snake identification and/or venomspecific enzyme immunoassay.

Main outcome measures: Clinical and laboratory features of envenoming.

Results: There were 24 confirmed cases of RSS envenoming, nearly all occurring in coastal areas between northern New South Wales and south-eastern Queensland. Twenty-three patients had local bite-site effects and 17 had at least three non-specific systemic effects (eg, nausea, headache). All 24 had venom-induced consumption coagulopathy (VICC), and 19 had an international normalised ratio $>3.0$. Six had bleeding from the bite site or intravenous cannula site, 10 had blood detected on urinalysis, and one had a major intra-abdominal haemorrhage. Mild neurotoxicity developed in two patients, and one patient developed myotoxicity with generalised myalgia, myoglobinuria and a peak creatine kinase level of $59700 \mathrm{IU} / \mathrm{L}$. Twenty-three patients were treated with antivenom ( 21 with tiger snake antivenom, two with polyvalent antivenom). Free venom was undetectable in 19 of 20 blood samples taken after antivenom administration.

Conclusion: RSS envenoming occurs predominantly in coastal areas of northern NSW and southern Queensland, and within this range, most envenoming is due to the RSS rather than tiger snakes. Clinically it is characterised by VICC, with mild neurotoxicity and myotoxicity in some cases. Tiger snake antivenom appears to be effective against RSS envenoming.

\section{The rough-scaled snake}

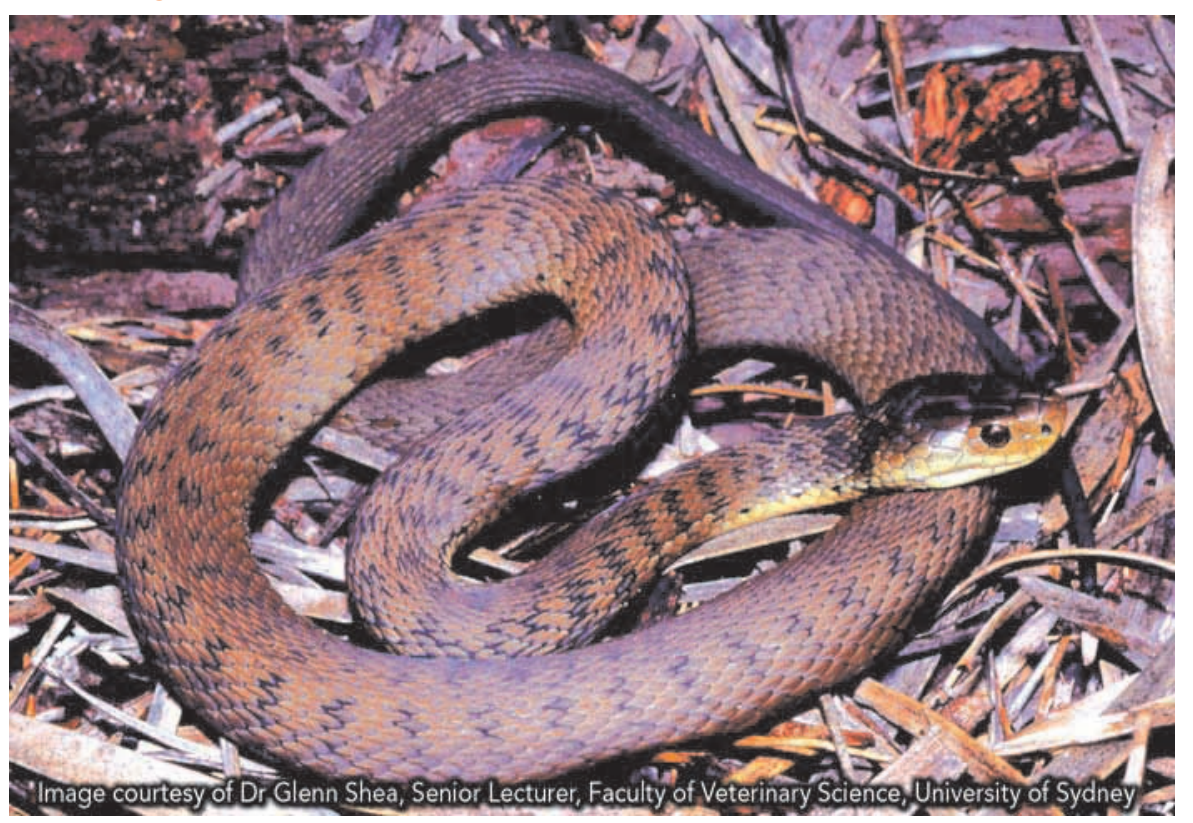




\section{Flowchart showing the sample used in our study}

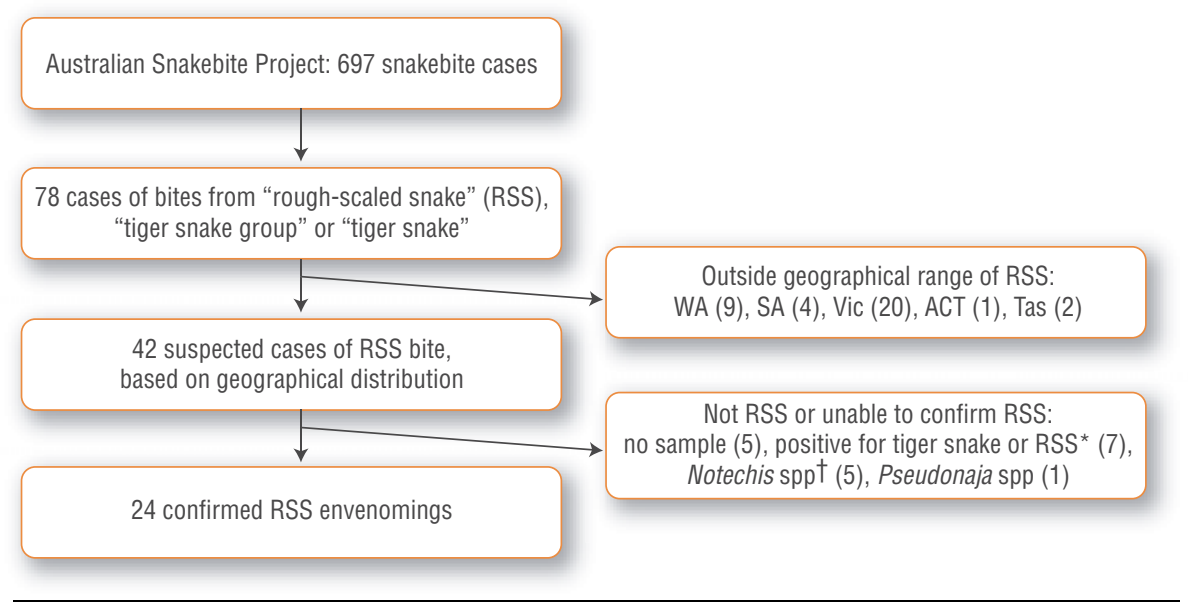

$\mathrm{ACT}=$ Australian Capital Territory. $\mathrm{SA}=$ South Australia. Tas $=$ Tasmania. Vic $=$ Victoria. $\mathrm{WA}=$ Western Australia. * Sample positive for venom from either Notechis spp (tiger snake) or Tropidechis carinatus (RSS) but below the limit of venom detection to distinguish between the two venoms. †Three cases involving identified Notechis spp biting snake handlers and two cases in southern New South Wales outside the geographical range of $T$. carinatus.

The aims of our study were to determine the occurrence and features of RSS envenoming using expert identification of the snake or EIA confirmation of venom in patient sera.

\section{METHODS}

\section{Patient cohort}

We analysed a cohort of patients from northern NSW and Queensland with potential RSS bites or envenoming — defined by snake identification, a positive SVDK result in the tiger snake well, or any clinical or laboratory features of elapid envenoming without a diagnostic SVDK result. The cohort was drawn from participants recruited into the Australian Snakebite Project (ASP). ${ }^{4}$ The ASP is an ongoing multicentre prospective observational study that recruits suspected snakebite and snake envenoming cases from over 100 major tertiary and regional hospitals and associated major poisons information centres. Ethics approval has been obtained from 16 human research ethics committees relevant to all institutions involved.

Demographic details, clinical effects and laboratory information and treatments relating to all patients recruited to the ASP are recorded on datasheets made available to the treating doctors. All data are entered into a purpose-designed relational database. Patients recruited to the ASP have serial serum samples collected for venom and antivenom quantification. Samples are spun, aliquoted, frozen and stored at $-80^{\circ} \mathrm{C}$ before analysis.

From all patients recruited to the ASP between January 2004 and December 2008, a cohort was identified by searching the database for "rough-scaled snake", "tiger snake group" and "tiger snake". Suspected tiger snake bites from areas clearly outside the range of $T$. carinatus (ie, Western Australia, South Australia, Tasmania and Victoria) were excluded, as well as cases in which there was a definite identification of a tiger snake or a member of the genus Hoplocephalus (Box 2). Finally, cases could only be included if there was a blood sample available from the patient before antivenom was administered or if the snake had been brought in for identification. Definite RSS envenoming was then confirmed by EIA and/or identification of the snake by a qualified expert. ${ }^{5}$

\section{Testing procedure}

EIA was performed on stored pre-antivenom serum samples (or plasma samples, if no serum was available), using a technique described in detail elsewhere. ${ }^{6}$ In brief, polyclonal antibodies (IgG) to RSS, common tiger snake (N. scutatus) and Stephens' banded snake (H. stephensii) were raised in rabbits, conjugated to biotin and used to develop a sandwich EIA using streptavidinhorseradish peroxidase as the detecting agent. The limits of detection were $0.3 \mathrm{ng} / \mathrm{mL}$ for the anti-RSS assay and $0.7 \mathrm{ng} / \mathrm{mL}$ for the anti-tiger snake assay. There was minimal cross-reactivity between antibodies to $H$. stephensii venom and antibodies to both RSS and tiger snake venoms. There was moderate cross-reactivity between RSS and tiger snake venoms and antibodies, but standard curves for each of these venoms did not overlap within the variability of the assay above $2 \mathrm{ng} / \mathrm{mL}$. For concentrations over $2 \mathrm{ng} / \mathrm{mL}$, RSS and tiger snake venoms could be accurately distinguished. Each sample was tested for all three venoms.

\section{Demographic, laboratory and clinical data}

Data on people with confirmed RSS envenoming were extracted from the ASP database. Information extracted included age, sex, geographical location, snake identification, SVDK results, clinical effects, laboratory investigations and antivenom treatment. Symptoms and signs were classified as follows:

- Local bite effects: pain, swelling or bruising at the bite site.

- Non-specific systemic effects: nausea, vomiting, headache, abdominal pain, diarrhoea or diaphoresis.

- VICC. This was defined as evidence of complete consumption coagulopathy, indicated by either an undetectable fibrinogen level or a raised D-dimer level (at least 10 times above the assay cut-off point or $>2.5 \mathrm{mg} / \mathrm{L}$ ) associated with an international normalised ratio (INR) $>3.0$. Mild VICC was defined as incomplete consumption coagulopathy, characterised by a low but detectable fibrinogen level or a raised Ddimer level (above the assay cut-off point) associated with a maximum INR between 1.5 and 2.5 .

- Neurotoxicity. This was defined by ptosis, external ophthalmoplegia, bulbar palsy, respiratory muscle paralysis or limb paralysis.

- Myotoxicity. This was defined by local or generalised myalgia and/or muscle weakness in association with a creatine kinase level > 1000 IU/L (reference range [RR], 0-195 IU/L).

- Leukocytosis and lymphopenia. Leukocytosis was defined as a white cell count $>11.0 \times 10^{9} / \mathrm{L}\left(\mathrm{RR}, 4.0-11.0 \times 10^{9} / \mathrm{L}\right)$, and lymphopenia as a lymphocyte count $<1.0 \times 10^{9} /$ L (RR, $\left.1.0-4.0 \times 10^{9} / \mathrm{L}\right)$.

\section{Statistical analysis}

For descriptive statistics, median and interquartile range (IQR) were calculated for data that were not normally distributed. 


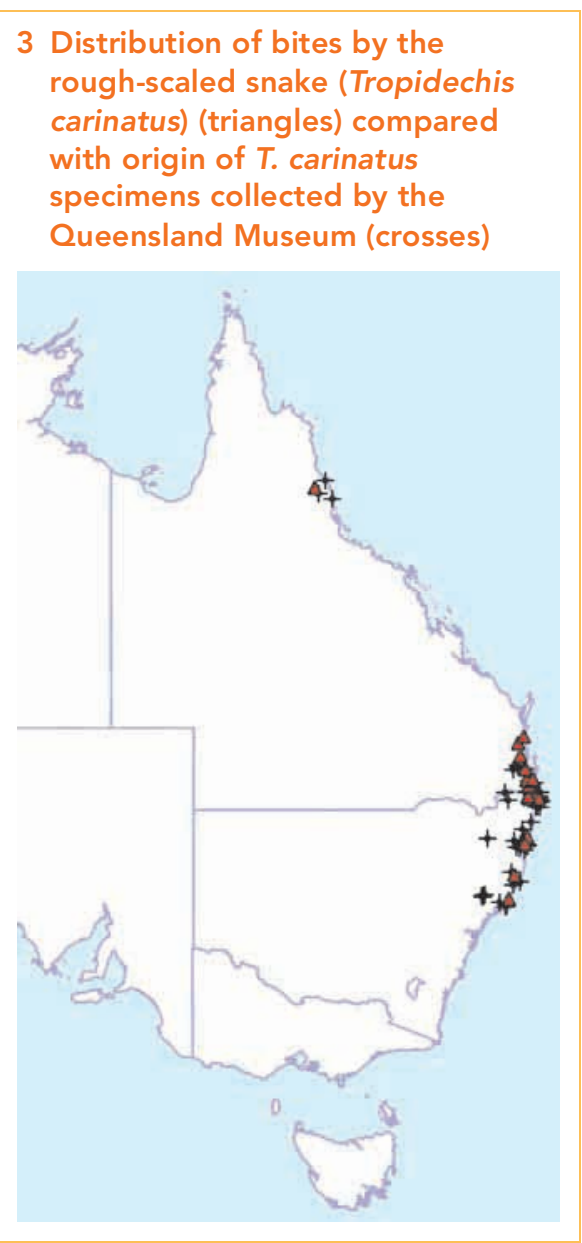

\section{RESULTS}

Of 697 people with snakebite recruited to the ASP, 42 were identified as potential RSS bite victims based on geographical location, SVDK results and clinical effects. Of these 42 people, 24 were confirmed to have experienced RSS envenoming, based on EIA or definite snake identification (Box 2). EIA results were available for 23 patients (median venom concentration, $17 \mathrm{ng} / \mathrm{mL}$ [IQR, 15-32 ng/mL]). The snake was identified and venom detected in serum in three cases. In one case, confirmation was based on snake identification alone, as no preantivenom serum sample was available.

All RSS bites investigated in our study occurred between Newcastle (mid-eastern NSW) and the Sunshine Coast (south-eastern Queensland), except for one isolated case in Cairns (northern Queensland) (Box 3). The median age of the 24 patients was 35 years (IQR, 21-49 years; range, 9-60 years) and 21 of the 24 patients were male. Based on bite-site swabs tested with SVDK, 20 cases were positive for tiger snake venom, two were negative and one was equivocal (in one case, the bite-site SVDK test was not done). Of four urine samples tested by SVDK, all were positive for tiger snake.

Twenty-three patients complained of local bite effects, and 17 reported three or more non-specific systemic effects (Box 4). In the 19 patients who had VICC, recovery of INR to 2.0 or less took a median time of 11.5 hours (IQR, 10.0-13.4 hours). The remaining five patients had mild VICC. Six patients complained of either bleeding from the bite site or from an intravenous cannula site, and 10 had blood detected on urinalysis. One patient had an intra-abdominal haemorrhage requiring blood transfusion but not surgical intervention. Factor studies demonstrated specific deficiencies in fibrinogen, factor V and factor VIII, and partial reduction in factor II.

Neurotoxicity occurred in only two patients and was mild, with only ptosis developing. One of these patients also developed significant myotoxicity with generalised myalgia and myoglobinuria. His creatine kinase level peaked at $59700 \mathrm{IU} / \mathrm{L}$, but he did not develop renal impairment. Another two patients had a small rise in creatine kinase level, peaking at $1790 \mathrm{IU} / \mathrm{L}$ in both cases. Local myalgia was reported in a further three patients and generalised myalgia in another two. The only other significant laboratory results were leukocytosis in 22 patients, of whom 12 had lymphopenia. One patient had lymphopenia in the absence of leukocytosis. There were no deaths, and all patients were discharged from hospital without sequelae.

Twenty-three patients were treated with antivenom (21 with tiger snake antivenom, two with polyvalent antivenom; total median dose, 3 vials [IQR, 2-4 vials]), and two patients received fresh frozen plasma. Post-antivenom blood samples were available for 20 of the 23 patients receiving antivenom. In 19 of these patients, no free venom was detected after a median first dose of 2 vials (IQR, 1.5-4 vials), and in five patients, free venom was undetectable after administration of only 1 vial. In one patient who was given only a small amount of 1 vial (due to an allergic reaction), venom was still detectable after antivenom had been given.

\section{DISCUSSION}

The clinical effects of RSS envenoming included VICC, which was severe in most cases and associated with non-specific systemic symptoms - most commonly headache, nausea and abdominal pain. Major bleeding occurred in one case, and signifi-

\begin{tabular}{|c|c|}
\hline \multicolumn{2}{|c|}{$\begin{array}{l}4 \text { Clinical features of rough-scaled } \\
\text { snake envenoming }\end{array}$} \\
\hline Clinical feature & $\begin{array}{l}\text { Number } \\
\text { of cases }\end{array}$ \\
\hline $\begin{array}{l}\text { Bite-site swab positive for tiger } \\
\text { snake venom using SVDK }\end{array}$ & 20 \\
\hline Local bite-site effects & 23 \\
\hline Pain & 23 \\
\hline Swelling & 16 \\
\hline Bruising & 9 \\
\hline $\begin{array}{l}\text { Three or more non-specific } \\
\text { systemic symptoms }\end{array}$ & 17 \\
\hline Nausea & 21 \\
\hline Vomiting & 12 \\
\hline Headache & 20 \\
\hline Abdominal pain & 14 \\
\hline Diarrhoea & 2 \\
\hline Diaphoresis & 7 \\
\hline VICC or mild VICC & 24 \\
\hline INR $>3.0$ & 19 \\
\hline External bleeding & 6 \\
\hline Bleeding from bite site & 3 \\
\hline $\begin{array}{l}\text { Bleeding from intravenous } \\
\text { cannula site }\end{array}$ & 3 \\
\hline Intra-abdominal bleed & 1 \\
\hline Blood detected on urinanalysis & 10 \\
\hline Neurotoxicity & 2 \\
\hline Myotoxicity & 3 \\
\hline Leukocytosis & 22 \\
\hline Lymphopenia & 13 \\
\hline
\end{tabular}

INR = international normalised ratio. SVDK = snake venom detection kit. VICC = venom-induced consumption coagulopathy.

cant myotoxicity along with mild neurotoxicity occurred in only one case.

RSS venom contains presynaptic and postsynaptic neurotoxins, myotoxins and a prothrombin activator that causes VICC. ${ }^{1}$ The RSS prothrombin activator is structurally and functionally similar to human factor $\mathrm{Xa}$ and belongs to the same group of prothrombin activator toxins as those produced by Notechis and Hoplocephalus spp. ${ }^{7}$

Most previous reports of RSS envenoming have been anecdotal cases involving identified snakes or cases occurring within the range of the RSS and shown to be SVDKpositive for tiger snake venom, and thus assumed to be the result of RSS bites. Trinca and colleagues ${ }^{2}$ reported 12 confirmed RSS bites in humans, but provided limited details on the clinical effects. Of the 12 patients, one died within 5 minutes of the 
bite and nine had non-specific systemic symptoms, including headache, nausea, vomiting, abdominal pain and diaphoresis. There was no information on whether coagulopathy occurred, and insufficient clinical detail to determine whether significant myotoxicity or neurotoxicity occurred. Almost no laboratory testing was done.

Other reports include possible RSS bites that occurred within the known range of the snake and were positive for tiger snake venom with SVDK. ${ }^{3,8,9}$ Other larger series of snake envenoming from Queensland are likely to include cases involving T. carinatus, but these cannot be distinguished from other cases involving snakes testing positive for tiger snake venom with SVDK. ${ }^{10-12}$ Myotoxicity and neurotoxicity have been reported in all these cases, but without confirmation by snake identification they provide limited useful information on RSS envenoming.

The clinical and laboratory effects of RSS envenoming appear to be similar to those of tiger snake envenoming. ${ }^{13,14}$ Although our series was too small to determine whether RSS envenoming is similar in the severity and frequency of its effects, all major effects reported in tiger snake envenoming occurred in our series of RSS bites, including VICC, myotoxicity and neurotoxicity.

In our study, SVDK testing of bite-site swabs was positive for tiger snake venom in nearly all RSS cases, confirming that RSS venom gives a positive result for tiger snake with SVDK. Conversely, of the cases of snake envenoming occurring within the geographical range of RSS and testing positive for tiger snake venom with SVDK, the majority ( 24 of 31 cases) were in fact RSS envenoming cases (Box 2).

The measurement of venom concentrations in patient serum samples allowed us to determine whether antivenom was effective in binding all venom that was present. We found that tiger snake antivenom binds RSS venom and appears to be effective in patients, even at lower doses than currently recommended.

A limitation of our study was that we could only include envenomed patients in the cohort because the venom assay relies on the detection of venom in blood. The study is therefore likely to be biased towards more severe cases, and we were unable to establish the envenoming rate for RSS (ie, number of cases of envenoming divided by number of definite bites).

Our study suggests that most, and potentially all, tiger snake SVDK-positive cases in northern NSW and south-eastern Queensland are likely to be due to RSS and that the envenoming syndrome is similar to that of Notechis spp. Tiger snake antivenom appears to bind all venom, even after administration of a single vial.

\section{ACKNOWLEDGEMENTS}

We wish to acknowledge the many clinicians and laboratory staff involved in recruiting and collecting data for the ASP and, in particular, for patients recruited to our cohort by Robert Bonnin, Mark Coghlan, David Ward, Andrew Parkin, Colin Page and Julian White. We also thank clinical toxicologists at poisons information centres around Australia for referring additional cases, and acknowledge the help of many nurses, doctors and laboratory staff in recruiting patients and collecting samples. We thank the Hunter Haematology Research Group for doing the clotting factor assays. Our study was supported in part by a National Health and Medical Research Council (NHMRC) project grant. Geoffrey Isbister is supported by an NHMRC clinical career development award and Simon Brown by an NHMRC career development award.

\section{COMPETING INTERESTS}

None identified.

\section{AUTHOR DETAILS}

Melissa Gan, MB BS, Emergency Registrar ${ }^{1}$ Margaret A O'Leary, PhD, Hospital Scientist ${ }^{2}$ Simon G A Brown, MB BS, FACEM, PhD, Professor, and Emergency Physician ${ }^{3,4,5}$ Tamara Jacoby, BSc, PhD Student ${ }^{3,4}$

David Spain, MB BS, FACEM, Emergency Physician $^{1}$

Alan Tankel, FACEM, Director and Emergency Physician $^{6}$

Chris Gavaghan, MB BS, FACEM, Emergency Physician $^{7}$

Peter Garrett, MB BS, FACEM, Intensive Care Physician $^{8}$

Geoffrey K Isbister, BSc, FACEM, MD, Principal Research Fellow and Clinical Toxicologist ${ }^{9}$

1 Emergency Department, Gold Coast Hospital, Gold Coast, QLD.

2 Department of Clinical Toxicology and Pharmacology, Calvary Mater Hospital, Newcastle, NSW.

3 Centre for Clinical Research in Emergency Medicine, Western Australian Institute for Medical Research, Perth, WA.

4 Centre for Medical Research, University of Western Australia, Perth, WA.

5 Department of Emergency Medicine, Royal Perth Hospital, Perth, WA.

6 Emergency Department, Coffs Harbour Base Hospital, Coffs Harbour, NSW.

7 Emergency Department, Lismore Base Hospital, Lismore, NSW.

8 Intensive Care Unit, Nambour Base Hospital, Nambour, QLD.
9 Tropical Toxinology Unit, Menzies School of Health Research, Charles Darwin University,

Darwin, NT.

\section{Correspondence:}

geoffrey.isbister@menzies.edu.au

\section{REFERENCES}

1 Department of Toxinology, Women's and Children's Hospital, Adelaide. Tropidechis carinatus. http://www.toxinology.com/fusebox.cfm? fuseaction=main. snakes. display\&id=SN0536 (accessed Aug 2008).

2 Trinca JC, Graydon JJ, Covacevich J, Limpus C. The rough-scaled snake (Tropidechis carinatus): a dangerously venomous Australian snake. Med J Aust 1971; 2: 801-809.

3 Jolles S, Lawrence S, Gallagher J, Fulcher D. Severe rhabdomyolysis after tiger snake bite. J R Soc Med 1998; 91: 267-269.

4 Isbister GK, Brown SG, MacDonald E, et al. Current use of Australian snake antivenoms and frequency of immediate-type hypersensitivity reactions and anaphylaxis. Med J Aust 2008; 188: 473-476.

5 O'Leary MA, Isbister GK, Schneider JJ, et al. Enzyme immunoassays in brown snake (Pseudonaja spp.) envenoming: detecting venom, antivenom and venom-antivenom complexes. Toxicon 2006; 48: 4-11.

6 O'Leary MA, Brown SGA, Jacoby $T$, et al. A sensitive and specific enzyme immunoassay to distinguish between two closely related Australian elapids: Tropidechis carinatus and Notechis scutatus. 8th International Society on Toxinology Asia-Pacific Meeting on Animal, Plant and Microbial Toxins; 2008 Dec 2-6; Hanoi and Halong Bay, Vietnam. IST, 2008.

7 Joseph JS, Chung MC, Jeyaseelan K, Kini RM. Amino acid sequence of trocarin, a prothrombin activator from Tropidechis carinatus venom: its structural similarity to coagulation factor Xa. Blood 1999; 94: 621-631.

8 Patten BR, Pearn JH, DeBuse P, et al. Prolonged intensive therapy after snake bite: a probable case of envenomation by the roughscaled snake. Med J Aust 1985; 142: 467-469.

9 Gavaghan CF, Sparkes G. Delayed myotoxicity in snake envenoming by the tiger snake group. Emerg Med (Fremantle) 2003; 15: 497-499.

10 Barrett R, Little M. Five years of snake envenoming in far north Queensland. Emerg Med (Fremantle) 2003; 15: 500-510.

11 Munro JG, Pearn JH. Snake bite in children: a five year population study from South-East Queensland. Aust Paediatr J 1978; 14: 248-253.

12 Jamieson R, Pearn J. An epidemiological and clinical study of snake-bites in childhood. Med J Aust 1989; 150: 698-702.

13 White J. Clinical toxicology of snakebite in Australia and New Guinea. In: Meier J, White J, editors. Handbook of clinical toxicology of animal venoms and poisons. New York: CRC Press, 1995: 595-618.

14 White J, Duncan B, Wilson C, et al. Coagulopathy following Australian elapid snakebite: a review of 20 cases. In: Recent advances in toxinology research. Singapore: Venom and Toxin Research Group, National University of Singapore, 1992: 337-344.

(Received 1 Nov 2008, accepted 16 Apr 2009) 